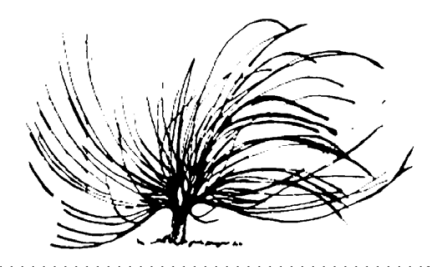

\title{
Alerta temprana: innovación educativa para disminuir el riesgo de abandono escolar en el Colegio Nocturno de Pococí
}

\author{
Maynor Jiménez-Castro ${ }^{l}$ \\ Colegio Nocturno de Pococí \\ Costa Rica \\ mynor.jimenez@ucr.ac.cr
}

Marjorie Umaña Calderón ${ }^{2}$

Colegio Nocturno de Pococí

Costa Rica

marjorie.umana.calderon@mep.go.cr

\begin{abstract}
Resumen
En este artículo, se expone una innovación educativa para la disminución del abandono escolar a través de una estrategia denominada Alerta temprana, la cual consiste en el registro de una serie de aspectos que muestra el estudiante cuando está en riesgo de abandono del sistema educativo. La estrategia es parte del programa "Yo me apunto" implementado por el Ministerio de Educación Pública de Costa Rica, pero ha sido adaptada, por el Colegio Nocturno de Pococí (CNP), para utilizar las tecnologías móviles y la herramienta de formularios de Google, en las cuales los docentes reportan, principalmente, el ausentismo, mientras los orientadores de nivel dan seguimiento a los casos indicados. Este mecanismo
\end{abstract}

Recibido: 31 de enero de 2020. Aprobado: 2 de setiembre de 2020.

http://dx.doi.org/10.15359/rep.16-1.12

1 Máster en Ciencias de la Computación (ITCR) y Didáctica de la Matemática (UGR). En la actualidad doctorando en la Universidad de Granada-España (UGR), profesor en la Universidad de Costa Rica y el Colegio Nocturno de Pococí. https://orcid.org/0000-0001-7984-6866

2 Máster en Administración Educativa (UCA), subdirectora del Colegio Nocturno de Pococí y profesora de la Universidad de San José, Sede de Guápiles. https://orcid. org/0000-0001-8672-4787 
ha permitido identificar los principales factores de riesgo que presenta un estudiante antes de renunciar al colegio, lo que facilita la atención rápida y oportuna del caso y la toma de decisiones por parte de las autoridades institucionales. De esta forma, se describe la experiencia obtenida en el CNP, donde los índices de exclusión son muy altos y los resultados de aplicar esta estrategia evidencian una mejora significativa en la prevención y el seguimiento de los riesgos de abandono.

Palabras clave: abandono escolar, deserción escolar, educación de adultos, educación en Costa Rica.

\begin{abstract}
In this article, an educational innovation for the reduction of school dropout is described through a strategy called Early Warning, which consists of registering a series of aspects that the students show when they are at risk of dropping out from the educational system. The strategy is part of the Yo me apunto program implemented by the Costa Rican Ministry of Public Education, but has been adapted by the Pococí Adult Night High School (CNP) in order to use mobile technologies and the Google forms tool to make reports mainly of absenteeism by the teachers, while the group of school counselors follow each case up. This mechanism has allowed to identify the main risk factors that students present before leaving school, which makes it possible to provide prompt and timely attention to the cases and proper decision-making by institutional authorities. This article describes the experience obtained in the CNP, where the exclusion rates are very high, and the results of applying this strategy show a significant improvement in the monitoring and prevention of the risks of high school dropouts.
\end{abstract}

Keywords: Dropping out, school dropouts, adult education, education in Costa Rica 


\section{Introducción}

$\mathrm{E}$

1 Colegio Nocturno de Pococí (CNP) es una institución pedagógica para jóvenes y adultos, ubicado en la región Caribe Norte de Costa Rica; cuenta con 1034 estudiantes de escasos recursos económicos, quienes provienen de zonas rurales con grandes problemáticas sociales. Sin embargo, en el año 2017, se destacó como uno de los centros educativos nocturnos de mejor rendimiento académico en el circuito 01 de la Dirección Regional Educativa de Guápiles.

A pesar de lo anterior, desde el año 2013, el colegio ha experimentado niveles de deserción escolar que median el 33,6 \% de estudiantes al año. Ante esta problemática, en el 2014, se inició el proyecto "Forjando esperanzas", cuyo objetivo principal fue disminuir el abandono y aumentar el rendimiento académico (Jiménez-Castro, Denis y Vega, 2015).

Este proyecto contribuyó a sensibilizar a la población docente y administrativa de la entidad, lo que permitió adaptar la estrategia de Alerta temprana desarrollada por el Ministerio de Educación Pública de Costa Rica, a los requerimientos de una institución con altos índices de vulnerabilidad, como lo es el CNP. Dado lo anterior, en esta investigación se pretende identificar los principales factores que intervienen en el abandono escolar, con el fin de realizar una toma de decisiones acertada que permita intervenir, de manera oportuna, la problemática de la exclusión estudiantil en el Colegio Nocturno de Pococí.

En este artículo, se describe la implementación de una estrategia novedosa para disminuir el abandono estudiantil, por lo que se parte de una serie de antecedentes, en los cuales se presenta esta problemática en los centros educativos nocturnos del país y se detalla la forma en que el CNP ha desarrollado la estrategia, implementando una metodología de investigación-acción con la participación de 55 funcionarios de la institución y el análisis de 184 reportes de estudiantes en riesgo de deserción. Por último, se extrae una serie de conclusiones y retos que se plantean para el futuro.

\section{Referentes teóricos}

La educación académica nocturna es una modalidad del sistema educativo costarricense, creada para brindar acceso equitativo a jóvenes y adultos, que, por diversas razones, no han podido concluir sus estudios secundarios y desean completarlo (MEP, 2013b). 
Esta modalidad pedagógica se compone de un currículo básico de nueve materias, las cuales se ofrecen en horarios que van desde las seis de la tarde a las nueve y cincuenta de la noche; está especialmente diseñada para personas que trabajan o han tenido que abandonar el sistema educativo diurno y ven en esta alternativa una forma de concluir los estudios. Sin embargo, esta opción ha venido enfrentando serias limitaciones que afectan negativamente su desempeño, como es el rendimiento académico y el abandono estudiantil.

Según Barrantes (2014), de la Dirección de Gestión de Calidad del Ministerio de Educación Pública, esta es la modalidad educativa que presenta mayor exclusión en secundaria y eso tiene efectos negativos en las pruebas de bachillerato. Asimismo, se indica que el rendimiento académico en los colegios nocturnos, no promedia notas superiores a 66 en las pruebas de bachillerato formal desde el año 2005, cuando el rendimiento esperado debe ser igual o superior a 70 , lo que refleja un problema que se debe atender con urgencia.

Los datos del IV Informe del Estado de la Educación, así como los anuales sobre bachillerato y otras investigaciones, (Castillo, Chavarría y García, 2016; Dirección de Gestión y Evaluación de la Calidad, 2014, 2015, 2016; Programa Estado de la Nación, 2013), destacan una clara desventaja de los colegios nocturnos en comparación con los académicos diurnos, en relación con los porcentajes de promoción obtenidos en las pruebas de bachillerato formal.

De igual manera, en el aspecto del abandono estudiantil, los centros educativos de secundaria en Costa Rica, reportaron datos que establecieron promedios entre 10,2 \% y 10,7 \% durante los años 2010 y 2012; sin embargo, de acuerdo con el Programa Estado de la Nación (2013), se pudieron haber dado informes que minimizaran la deserción en estos periodos.

Dicha situación, en este mismo periodo, concuerda con el estudio realizado por el Proyecto de Apoyo a la Educación Secundaria para la Reducción del Abandono Escolar (ProEDUCA) (MEP, 2013a), el cual indica que la deserción estudiantil general pudo alcanzar en promedio $17,1 \%$ y la tasa en los colegios académicos nocturnos cerca de $32,5 \%$, lo cual demuestra que esta es la rama educativa con mayores problemas en ese ámbito.

Si bien como se indica en el Ministerio de Educación Pública (2016), el abandono escolar ha venido disminuyendo en los últimos 
años, por ejemplo, la exclusión intraanual reportada en el Programa Estado de la Nación (2018) para la educación académica nocturna en el año 2015, fue de un 25,7\%, inferior a la presentada en el Colegio Nocturno de Pococí para el mismo periodo, que fue de 32,8 \% (ver tabla 1).

Las causas de abandono escolar en Costa Rica han sido sujeto de análisis de distintas investigaciones, como se menciona en Jiménez y Gaete (2013); sin embargo, estas se han concentrado en situaciones particulares y no en indagaciones más amplias que aborden todo el sistema educativo costarricense, por lo que se considera lo aportado por MIDEPLAN (2007), en el cual esta problemática puede ser explicada con base en tres fenómenos: exclusión, expulsión y repulsión.

La exclusión se refiere al conjunto de situaciones extraescolares que imposibilitan al estudiante seguir en el proceso educativo (principalmente problemas económicos). La expulsión se refiere a circunstancias donde se destacan problemas de rendimiento académico o aprovechamiento, mientras que la repulsión a manifestaciones de desinterés de los alumnos por asistir a clases (poco arraigo con el grupo de compañeros, hostilidad de los docentes, entre otros).

En este mismo sentido, Espíndola y León (2002) consideran que el abandono escolar es:

...el resultado de un proceso en el que intervienen múltiples factores y causas, algunos de los cuales son característicos de los niños y jóvenes y de sus situaciones socioeconómicas (factores extraescolares) y de otros más asociados a las insuficiencias del propio sistema educativo (factores intraescolares). (p. 53)

El fracaso escolar y el bajo rendimiento, en los colegios académicos nocturnos, son solo dos aspectos que muestran las grandes asimetrías que presenta el sistema educativo costarricense. Los problemas sociales son parte de las causas que afectan las condiciones en las cuales se desarrolla la educación nocturna.

Lo anterior es reflejado en el IV Informe del Estado de la Educación (Programa Estado de la Nación, 2013), donde al analizar la génesis sobre abandono escolar, en un colegio de la región Caribe, se concluye que los problemas socioeconómicos del hogar, el acceso a las ayudas del Gobierno, la insatisfacción de los estudiantes con el apoyo que reciben de docentes y orientadores y la trayectoria académica del alumno 
(especialmente, si tiene un historial de repitencia), son los factores que más intervienen para que los discentes no permanezcan en las aulas.

Este análisis también concluye que "la probabilidad de que un alumno repitente de Limón sea excluido y se retire del colegio es casi cuatro veces mayor que la de un estudiante de la misma zona que no haya repetido" (Programa Estado de la Nación, 2013, p. 158).

Ante la problemática expuesta, el Ministerio de Educación Pública de Costa Rica, ha articulado esfuerzos con otros proyectos del gobierno, como lo son: Tejiendo desarrollo y Puentes de desarrollo, de manera que las ayudas y las orientaciones lleguen a las poblaciones más necesitadas y con riesgos de abandonar las aulas.

Para ello, se creó la estrategia: Yo me apunto, que facilita una serie de lineamientos ante los factores que afectan la exclusión estudiantil: ausentismo, bajas calificaciones, violencia, drogas, falta de interés, problemas económicos y familiares, entre otros, para que las autoridades educativas puedan intervenir con su respectivo plan remedial adaptado a las necesidades de cada estudiante (Programa Estado de la Nación, 2018).

Otra estrategia que surge de esta iniciativa es Alerta temprana, la cual se plantea como una herramienta para abordar riesgos como: ausentismo, problemas económicos, adicciones, desmotivación, entre otros, con el propósito de evitar que el estudiantado abandone sus estudios. Esta iniciativa facilita el registro las observaciones de los docentes para que, posteriormente, el departamento de orientación haga el análisis y el seguimiento respectivo.

\section{Estrategia metodología}

La implementación de la estrategia de Alerta temprana, en el Colegio Nocturno de Pococí, está ligada al proyecto: Forjando esperanzas, que inició en el año 2015 y que ha buscado herramientas para disminuir el abandono escolar y el mejorar los índices de rendimiento académico. Estos propósitos se han llevado a cabo siguiendo una metodología de investigación-acción, la cual ejecuta dos procesos en paralelo: por un lado, la investigación, que busca problematizar la realidad para asumirla como objeto de estudio, por otro lado, la acción, entendida como la posibilidad de generar un cambio social (Inciarte, 2011).

Esta metodología es normalmente entendida como intervencionista, pues dado un problema real se decide abordarlo con un marco 
teórico específico, el cual se traduce en acciones que se deberán implementar. Por su parte, el grupo involucrado en la investigación observa los resultados de las gestiones, reflexiona y vuelve a plantear nuevas alternativas.

De igual forma, la investigación acción participativa ha sido conceptualizada como "un proceso por el cual miembros de un grupo, o una comunidad oprimida, colectan y analizan información y actúan sobre sus problemas con el propósito de encontrarles soluciones y promover transformaciones políticas y sociales" (Selener, 1997, p. 17).

Sampieri, Collado y Baptista (2010), consideran que la investigación-acción cuenta con tres fases esenciales: (a) observación, en la cual se describe el problema y se recolectan datos que sustentan la situación; (b) reflexión, etapa en donde se analiza e interpreta el asunto y (c) actuación, donde se implementan acciones para resolver o mejorar la circunstancia detectada. Esto se realiza "de manera cíclica, una y otra vez, hasta que el problema es resuelto, el cambio se logra o la mejora se introduce satisfactoriamente" (Sampieri et al., 2010, p. 553).

En este sentido, se ha logrado la participación del cuerpo docente de la institución, el cual está compuesto por 47 profesionales de diferentes materias, seis orientadores y dos auxiliares administrativos, cuya labor consiste en registrar los datos de los estudiantes con riesgo de deserción, así como las acciones implementadas para retener, reinsertar o bien registrar las principales causas del abandono.

Las acciones se han realizado utilizando los formularios de Google Forms, los cuales son herramientas que dispone Google para aplicar encuestas o consultas simples, donde el registro, la tabulación y el análisis de los datos se realiza de manera fácil y sencilla.

Esta metodología se basó en un enfoque cualitativo; sin embargo, en la interpretación de la realidad se incorporaron elementos cuantitativos que facilitan el análisis descriptivo sobre los riesgos de abandono que intervienen en la población estudiantil del Colegio Nocturno de Pococí y que no se encuentran sistematizados para una toma de decisiones más acertada. Este proceso se puede visualizar en la figura 1. 


\section{Proceso para prevenir y}

reducir el abandono

estudiantil

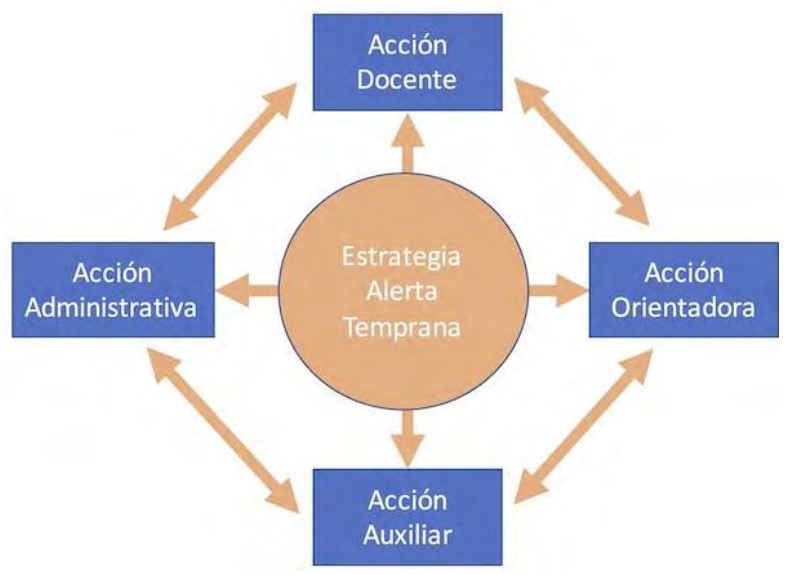

Figura 1. Proceso de atención ante el riesgo de abandono o reinserción estudiantil en el Colegio Nocturno de Pococí.

Nota: elaboración propia de los autores.

El modelo descrito en la figura 1, representa las interacciones que siguieron los reportes digitales desde el inicio con la intervención de los docentes, hasta el análisis realizado por la gestión administrativa de la institución. En este caso, se realizaron 184 registros de estudiantes en riesgo de abandono y se identificaron los principales factores que motivan la exclusión de los estudiantes del CNP.

\section{Proceso de implementación de la estrategia}

El Colegio Nocturno de Pococí, a través de varios años, ha presentado grandes problemas de repitencia y abandono escolar, lo que ha hecho que tanto el cuerpo docente como administrativo sienta la necesidad de investigar las causas de esto, a la vez que ha procurado atenuar dicha problemática, por lo que ha realizado un registro del nivel de deserción en los últimos cinco años, lo que se visualiza en la tabla 1: 
Tabla 1. Registro histórico de matrícula inicial y abandono estudiantil en el CNP (2013-2017)

\begin{tabular}{lccccc}
\hline \multicolumn{1}{c}{ Reporte Anual } & $\mathbf{2 0 1 3}$ & $\mathbf{2 0 1 4}$ & $\mathbf{2 0 1 5}$ & $\mathbf{2 0 1 6}$ & $\mathbf{2 0 1 7}$ \\
\hline Matrícula Inicial & 1744 & 1541 & 1355 & 1192 & 1019 \\
\hline Abandono & 618 & 514 & 444 & 414 & 325 \\
\hline$\%$ Abandono & $35,4 \%$ & $33,4 \%$ & $32,8 \%$ & $34,7 \%$ & $31,9 \%$ \\
\hline
\end{tabular}

Nota: datos propios del CNP.

Los datos reflejados en la tabla 1 , muestran la problemática de exclusión educativa en el periodo del año 2013 al 2017, la cual promedia el 33,64 \% de abandonos al año. Es importante tomar en cuenta que en el 2017 se muestra una disminución del fenómeno, lo cual coincide con la aplicación del plan piloto de la Alerta temprana, iniciado en el segundo semestre del 2017.

La estrategia para disminuir la deserción escolar consiste en un registro semanal, a través de un formulario de Google, en el cual acceden los docentes, para enviar la información del estudiante que presenta niveles reiterados de ausentismo. El profesor, al finalizar la semana lectiva, realiza el informe digital en el que se consignan los datos personales del alumno, la materia en la cual está faltando, el nivel y la sección a la que pertenece y las acciones realizadas (llamadas al hogar, consulta a compañeros, etc., que lo llevó a considerar al educando sujeto de seguimiento, o bien, reportarlo al orientador respectivo de nivel), esta información es almacenada en la nube, en una hoja de electrónica, la cual es accedida por el guía del nivel o de sección, para analizar cada caso reportado.

A partir de la información suministrada por el docente, el orientador del nivel evalúa los casos y da seguimiento, con base en lo cual procede a comunicarse con el estudiante o la familia de este para conocer los motivos de sus ausencias a clases. A partir de estas acciones, registra la información en otro formulario de Google, al cual tienen acceso los auxiliares (encargados de llevar al día el registro electrónico estudiantil), quienes facilitan la información a los docentes para que actualicen los registros estudiantiles.

Del mismo modo, la coordinación académica y la dirección institucional utilizan dichos datos para su análisis y toma de decisiones en la asignación de personal por nivel, reacomodo de grupos, estrategias para la retención estudiantil, asignación de becas especiales, traslados 
de estudiantes entre las aulas, charlas para atender las necesidades de los educandos, actividades extracurriculares, entre otros.

\section{Discusión de resultados}

La aplicación de la Alerta temprana, en el CNP, inicia en el mes de julio del 2017 con una población de 863 estudiantes, es decir, ya 156 educandos habían abandonado la institución, según lo establecido en la tabla 1. A partir de este momento, el personal pedagógico inicia el registro semanal del ausentismo estudiantil vía formularios de Google, el cual se extiende hasta el final del curso lectivo 2017. En este periodo, se realizaron 372 reportes, donde se destaca al III Ciclo de la Educación General Básica (sétimo, octavo y noveno) como el de mayor alerta de deserción con el 73,3\% de los reportes docentes. Con esta información, los orientadores de su respectivo nivel se dieron la tarea de indagar a profundidad sobre el ausentismo del estudiante, lo que refleja 184 casos atendidos, de los cuales se obtuvo la información de la figura 2 .

\section{Estudiante en riesgo de abandono}

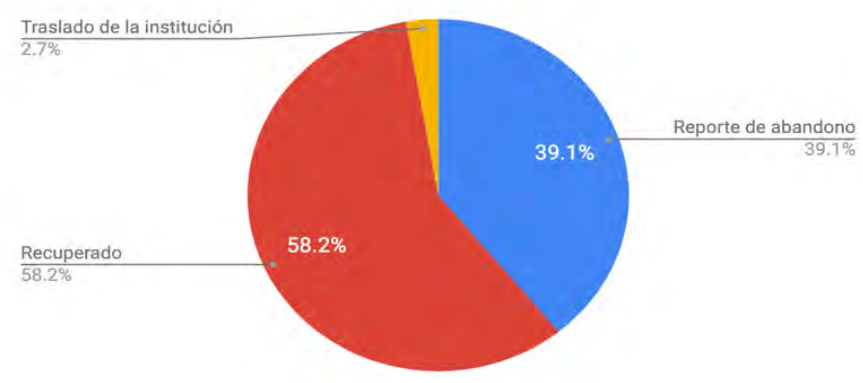

\section{Figura 2. Reportes de seguimiento realizado por los orientadores en el II semestre 2017.}

Nota: datos propios del CNP obtenidos de Google Forms.

En la figura 2, se muestra que el 58,2 \% de los casos reportados lograron ser reincorporados a la institución, es decir, 107 estudiantes se motivaron para que regresaran a clases. Igualmente, el 39,1\% de los discentes reportados no volvieron, es decir, 72 alumnos abandonaron la institución y $5(2,7 \%)$ realizaron gestiones de traslado a otros colegios. 
Por su lado, 29,2 \% de quienes dejaron el colegio, expresaron tener inconvenientes con cambios atinentes a lo laboral (cambio de trabajo, horarios, etc.) y el 15,3\% alegaron motivos personales ajenos al control de la institución. Los demás argumentos tienen que ver con situaciones variadas como: personales, económicas, desinterés por el estudio, dificultad para el cuido de sus hijos, entre otras. Por último, los casos de deserción reportados por los orientadores se lograron clasificar según el factor de abandono, como se muestra en la figura 3.

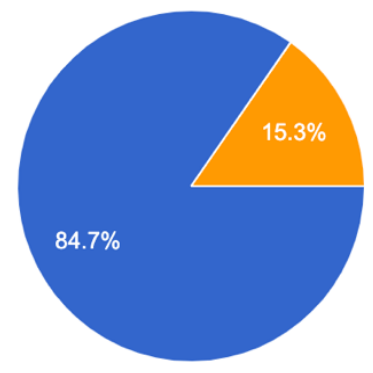

Exclusión: Asociada con factores socio económicos que impiden la permanencia de los estudiantes en el sistema educativo

Expulsión: Asociada con mecanismos inadecuados de evaluación, repitencia y reprobación

Repulsión: Relacionada con la falta de pertenencia, utilidad o interés desde la percepción de los y las estudiantes

\section{Figura 3. Clasificación del abandono según seguimiento de los orientadores en el II semestre 2017.}

Nota: datos propios del CNP, obtenidos del Google Forms.

E1 84,7\% de los educandos que abandonaron el colegio se debió a causas de exclusión, es decir, a situaciones socioeconómicas en las que la institución no puede hacer grandes esfuerzos. El restante 15,3\% se dio por factores de repulsión, lo que indica que el centro educativo debe buscar o promover actividades que fortalezcan el vínculo con el estudiante, es decir, clases más dinámicas, actividades recreativas, culturales y deportivas que permitan mejorar los niveles de pertenencia del discente en la academia.

Por su parte, la aplicación de la estrategia de Alerta temprana en el segundo trimestre del 2018, dejó algunos resultados importantes por destacar; 179 reportes docentes, en los cuales se remitieron más de 430 estudiantes como sujetos de análisis y seguimiento por el riesgo que presentaban de abandonar el colegio. Sin embargo, a pesar del esfuerzo realizado, 156 alumnos dejaron sus estudios y de 84 casos analizados, 
por los orientadores de nivel, solo 30 educandos (36,9\%) se reincorporaron al sistema.

De la misma manera que los datos del 2017, los principales factores que inciden en el abandono tienen que ver con la expulsión en un $84 \%$ y la repulsión con un $16 \%$. Cabe mencionar que, como consecuencia de la huelga histórica, puesta en marcha a partir del mes de setiembre del 2018 y finalizada en diciembre del mismo año, no se pueden visualizar los resultados reales de la aplicación de dicha estrategia (Programa Estado de la Nación, 2019). Esto por cuanto al estar la mayor parte del cuerpo docente, administrativo y técnico en paro, los estudiantes no fueron convocados al centro educativo durante esos tres meses.

\section{Conclusiones}

La estrategia de Alerta temprana, implementada en el Colegio Nocturno de Pococí, empieza a dar sus frutos y ha demostrado ser útil para el rescate y la reincorporación de estudiantes al sistema educativo. Esto se reflejó con una disminución de 2,8 \% de abandonos con respecto al año 2016.

De igual manera, la implementación de esta estrategia ha permitido conocer las principales causas que intervienen en la decisión final de abandonar el colegio, como lo son: problemas atinentes a situaciones laborales, económicas, cuido de los hijos, y desinterés por el estudio, lo que brinda la posibilidad de actuar de manera inmediata para atenuar la problemática que vive el estudiante.

Igualmente, esta estrategia ha permitido buscar alternativas para atender las causas de abandono antes mencionadas; en el caso de cambios de trabajo o las jornadas laborales, se ha realizado flexibilidad horaria y la asignación de tareas compensatorias, así como se proyecta a la implementación de aulas virtuales o bien espacios de interacción asincrónicos, donde los educandos puedan estar "conectados" con los estudios, aunque de manera remota y a su tiempo.

También, se plantea mejorar el apoyo económico con becas y otros servicios complementarios, esto para quienes han expresado situaciones financieras temporales. En el caso de estudiantes que tienen inconvenientes con el cuido de sus hijos, se coordinó una alianza con el Centro de Educación y Nutrición (CEN) y Centro Infantil de Nutrición y Atención Integral (CINAI) de Guápiles para dar prioridad a los estudiantes con dicha problemática. 
Asimismo, se proyecta la construcción de una guardería infantil dentro del CNP. Por último, se ha motivado a los docentes para hacer más atractiva la dinámica de clase, a través de la integración de tecnologías digitales y la creación de espacios de socialización dentro y fuera del aula.

Por último, la estrategia de Alerta temprana también ha generado grandes retos para contar con información inmediata y así, evitar el avance en el riesgo de abandono por parte de los discentes, lo cual plantea la posibilidad de incorporar otros actores en el sistema de alerta, como lo pueden ser estudiantes líderes de aula que reporten de manera más ágil y efectiva el riesgo de deserción escolar.

\section{Reconocimientos}

Se hace un reconocimiento especial a todos los docentes, los auxiliares administrativos y los orientadores del Colegio Nocturno de Pococí, que de manera constante han participado en el proceso de implementación de la Alerta temprana, pues sin su apoyo, la disminución en el abandono estudiantil no se habría logrado.

\section{Referencias}

Barrantes, A. (26 de noviembre de 2014). Colegios nocturnos sacan las peores notas en bachillerato. La Nación. Recuperado de http:// www.nacion.com/nacional/educacion/Colegios-nocturnos-sacan-peores-bachillerato_0_1453654659.html

Castillo, M., Chavarría, J. M. y García, M. (2016). Rendimiento académico en las pruebas nacionales de matemática en colegios del área metropolitana y zonas alejadas de Costa Rica en 2013. UNICIENCIA, 30(1), 85-97. https://doi.org/http://dx.doi. org/10.15359/ru.30-1.5

Dirección de Gestión y Evaluación de la Calidad. (2014). Bachillerato de la educación formal 2013: rendimiento y niveles de desempeño. San José, Costa Rica, Ministerio de Educación Pública.

Dirección de Gestión y Evaluación de la Calidad. (2015). Bachillerato de la educación formal 2014: rendimiento y niveles de desempeño. San José, Costa Rica, Ministerio de Educación Pública.

Dirección de Gestión y Evaluación de la Calidad. (2016). Bachillerato de la educación formal 2015: rendimiento y niveles de desempeño. San José, Costa Rica, Ministerio de Educación Pública. 
Espíndola, E. y León, A. (2002). La deserción escolar en América Latina: un tema prioritario para la agenda regional. Revista Iberoamericana de Educación, 30, 39-62. Recuperado de http:// www.seg.guanajuato.gob.mx/Ceducativa/CartillaB/6antologia/ antecedentes/pdf/32.-\%20LA\%20DESERCI\%C3\%93N\%20 ESCOLAR\%20EN\%20AM\%C3\%89RICA\%20LATINA\%20 UN\%20TEMA\%20PRIORITARIO\%20PARA\%20LA\%20 AGENDA\%20REGIONAL.pdf

Inciarte, A. (2011). Investigación acción: metodología transformadora. Utopía y Praxis Latinoamericana, 16(52), 131-138.

Jiménez-Castro, M., Denis, S. y Vega, W. (2015, 26-28 de marzo). Forjando esperanzas en los colegios nocturnos del Caribe Norte de Costa Rica [Presentación de ponencia]. Memorias XIV Jornadas y II Congreso Internacional del Maestro Investigador. Universidad Pontificia Bolivariana, Medellín, Colombia.

Jiménez, W. y Gaete, M. (2013). Estudio de la exclusión educativa y abandono en la enseñanza secundaria en algunas instituciones públicas de Costa Rica. Revista Electrónica Educare, 17(1), 105-128. https://doi.org/10.15359/ree.17-1.6

MEP (2013a). Abandono escolar en la secundaria costarricense: estudio de línea de base para los 100 centros educativos del ProEDUCA, 2012 (1 ed.). San José, Costa Rica: Fondo de las Naciones Unidas para la Infancia.

MEP (2013b). Educación de personas jóvenes y adultas. Recuperado el 5 de enero del 2015 de http://www.mep.go.cr/ educacion-de-personas-jovenes-y-adultas

MIDEPLAN (2007). Plan Nacional de Desarrollo "Jorge Manuel Dengo Obregón” 2006-2010. San José, Costa Rica: Autor.

Ministerio de Educación Pública. (2016). Exclusión educativa en el sistema público costarricense (análisis de cinco dimensiones). San José, Costa Rica, Ministerio de Educación Pública.

Programa Estado de la Nación (2013). Estado de la Educación Costarricense. IV Informe del Estado de la Educación. San José, Costa Rica.

Programa Estado de la Nación (2018). Estado de la Educación Costarricense. Sexto Informe del Estado de la Educación. San José, Costa Rica. 
Programa Estado de la Nación. (2019). Estado de la Educación Costarricense. Séptimo informe del Estado de la Educación. San José, Costa Rica.

Sampieri, R., Collado, C. y Baptista, M. del P. (2010). Metodología de la Investigación. (J. Mares Chacón, Ed.) (5. ${ }^{a}$ ed.). México, D. F.: McGrawHill Educación.

Selener, D. (1997). Participatory action research and social change (2. ${ }^{\mathrm{a}}$ ed.). NY: The Cornell Participatory Action Research Network, Cornell University. 
